\title{
What Can Be Improved in GC-MS-When Multi Benefits Can Be Transformed into a GC-MS Revolution
}

\author{
Aviv Amirav ${ }^{1,2 *}$, Alexander B. Fialkov ${ }^{1}$, Tal Alon ${ }^{1,2,3}$ \\ ${ }^{1}$ School of Chemistry, Tel Aviv University, Tel Aviv, Israel \\ ${ }^{2}$ Aviv Analytical Ltd, Tel Aviv, Israel \\ ${ }^{3}$ Afeka College, Tel Aviv, Israel \\ Email: *amirav@tau.ac.il
}

Received July 13, 2013; revised August 20, 2013; accepted September 15, 2013

Academic Editor: Ilia Brondz ${ }^{1,2}$

${ }^{1}$ Department of Biosciences, University of Oslo, Oslo, Norway; ${ }^{2}$ R\&D Department, Jupiter Ltd.

Copyright (C) 2013 Aviv Amirav et al. This is an open access article distributed under the Creative Commons Attribution License, which permits unrestricted use, distribution, and reproduction in any medium, provided the original work is properly cited.

\begin{abstract}
Gas chromatography mass spectrometry (GC-MS) is a widely used central analytical technology. Commercially available GC-MS systems use different types of mass analyzers such as Quadrupole, Ion Trap and/or Time of Flight, but practically all systems utilize the same Nier type electron ionization (EI) ion source and the same standard GC-MS transfer-line interface. Consequently, the various GC-MS vendors characterize their systems by a short list of specifications that relate to improvements in the technology of GC or of MS and not of the interfacing technology and ion source. This article presents a list of 62 ways in which the performance of GC-MS as a whole can be improved by an innovative interface and ion source. Such an interface can possibly lead to a GC-MS revolution in a way that is similar to that which is brought to Liquid Chromatography Mass Spectrometry (LC-MS) by the Electrospray ionization interface and ion source and not by improvements to the technology of LC or MS. These 62 possible GC-MS improvements (grouped into eight main categories) are not merely theoretical as they are provided by the Cold-EI GC-MS interface, which is based on the ionization of vibrationally cold sample molecules in a Supersonic Molecular Beam (SMB) within a flythrough ion source. An explanation and discussion is provided for each of these possible improvements.
\end{abstract}

Keywords: Gas Chromatography Mass Spectrometry (GC-MS); Supersonic Molecular Beams; Cold EI; Instrument Development

\section{Introduction}

GC-MS vendors typically describe and characterize their systems using a small set of specifications that include octafluoronaphthalene (OFN) signal to noise ratio (SNR), mass range and scan speed. As a result, improvements in GC-MS are often focused on the incremental increase of these few specifications. However, GC-MS is characterized by many additional features and operational parameters that contribute to its performance, and their improvements can make a big impact on the GC-MS analytical capabilities. Many such GC-MS aspects are improved by incorporating the new Cold-EI GC and MS interface and ion source technology and by using "out of the box" thinking. In LC-MS, the biggest revolution was brought not by LC or MS improvements but rather by the

\footnotetext{
"*Corresponding author.
}

development of a new interface and ionization method namely Electrospray. Similarly, Cold EI with its supersonic molecular beams interface and fly-through ion source brings multiple benefits and improvements into GCMS which can initiate a new GC-MS revolution. This manuscript lists 62 GC-MS improvements brought forth by the Cold EI interface and ion source, encompassing any and every important aspect of GC-MS, and explains how the unique features of GC-MS with Cold EI enable these benefits. When these multiple benefits come together, they amount to a whole new GC-MS experience that we believe is destined to ignite the next GC-MS revolution.

\section{5975-SMB GC-MS with Cold EI-The Technology and System}

GC-MS with Cold EI is based on the use of a Supersonic Molecular Beam (SMB) as an interface between the GC 
and MS and as a medium for the ionization of sample compounds while they are cold in the SMB. Thus, the Electron Ionization (EI) of cold molecules in SMB was termed as "Cold EI". Within this interface a supersonic molecular beam is formed by the expansion of a gas through a $\sim 0.1 \mathrm{~mm}$ pinhole into a vacuum chamber. During the expansion process the carrier gas (helium) and heavier sample molecules obtain the same final velocity so that the sample compounds are accelerated to the carrier gas velocity, since it is the major gas mixture component. This uniform velocity ensures slow intrabeam relative motion, resulting in the cooling of the sample compound internal vibrational degrees of freedom. SMB's are characterized by the following features of importance for mass spectrometry and GC-MS:

1) Super-cooling of the sample molecular vibrationalrotational degrees of freedom.

2) Hyperthermal sample molecular kinetic energy (up to $20 \mathrm{eV}$ ).

3) Unidirectional motion in space with heavy species concentration along the beam axis (jet separation).

4) High column flow rate compatibility up to $100 \mathrm{ml} / \mathrm{min}$.

These unique properties of SMB, which improve GCMS and GC-MS with Cold EI are explored by Amirav, Gordin, Poliak, and Fialkov (2008) [1].

In Figure 1, a schematic diagram of GC-MS with Cold EI is shown with its supersonic molecular beam interface and fly-through ion source.

The basic standard GC-MS instrument modifications for its conversion into GC-MS with Cold EI include:

a) The analytical column of a conventional GC with unrestricted column type (ID), length and flow rate is connected to a supersonic nozzle via a heated transfer line and mixed with added helium make up gas (typically $60 \mathrm{ml} / \mathrm{min}$ ).

b) Sampling to the MS vacuum system is in the form of skimmed supersonic molecular beam, as the organic sample compounds expand with the added make up helium gas from the supersonic nozzle into a separately (differentially) pumped nozzle vacuum chamber;

c) The electron ionization ion source is modified to allow for unperturbed axial passage of the molecular beam (fly-through) with a high (typically $8 \mathrm{~mA}$ ) ionizing electron emission current;

d) A suitable 90 degrees ion mirror is added to suppress mass spectral noise, keep the mass analyzer clean and for minimizing the added bench space.

\section{What Can Be Improved in GC-MS?}

A list of 62 GC-MS improvements is presented below, categorized into eight separate sections. The list includes an explanation of the ways in which GC-MS with Cold EI can provide these many benefits and improvements via the unique properties of its interface and ion source.

One can clearly see that the list of possible GC-MS improvements is far greater than commonly perceived since most of these improvements are not mentioned in any vendor specification or in any other type of publication or paper.

\subsection{Improved Sample Identification}

The coupling of Mass Spectrometry with Gas Chromatography is aimed at improved samples identification and quantification versus GC-FID. While GC-MS excels in sample identification its performance can be significantly further improved in a few ways. These improvements, when combined (as in the 5975-SMB GC-MS with Cold EI) can provide significantly improved confidence level in sample identification:

1) Enhanced molecular ions

The molecular ion is the single most important mass spectral peak for sample identification. Standard EI produces molecular ions for about $70 \%$ of the samples and as the sample compound gets bigger its molecular ion relative abundance gets smaller. In Cold EI the molecular cooling reduces the internal vibrational energy and thus decreases the chance of fragmentation after the sample compound impact with an ionizing electron and as a result the molecular ion abundance is enhanced. The degree of Cold EI enhancement of the molecular ion is small for small and rigid compounds (enhancement factor of near 1) while for large compounds it can be more than 1000 times due to the large heat capacity of large polyatomic compounds. The molecular ion enhancement factor dependence on compound size was demonstrated and discussed by Amirav, Keshet, and Alon (2012) [2]. The standard alternative to EI for the provision of molecular ions is chemical ionization (CI), however, while CI (or APCI) provides molecular ions its response (ionization yield) is highly non uniform, certain compounds are not properly ionized with it, its mass spectra are incompatible with library search, the closed CI ion source induces peak tailing and sample decomposition even more than standard EI ion source, and it adds cost and requires venting for ion source replacement. Cold EI enhances the molecular ions which are provided for about $99 \%$ of the compounds combined with uniform ionization yield and without any of the CI downsides as above.

2) Improved confidence in the identity of the molecular ions.

While the molecular ion can be found in standard EI spectra, often it is weak and can not be trusted since it can be suspected to be an impurity or matrix ion, vacuum background or high mass sample fragment ion. In Cold EI the molecular ion can be trusted more than in standard EI. This is in part due to the enhancement of the relative abundance of the molecular ion and in part due to the elimination of vacuum background of ions with masses above the molecular ion. Thus, the highest mass spectral peak can be assumed with high confidence level to be the 


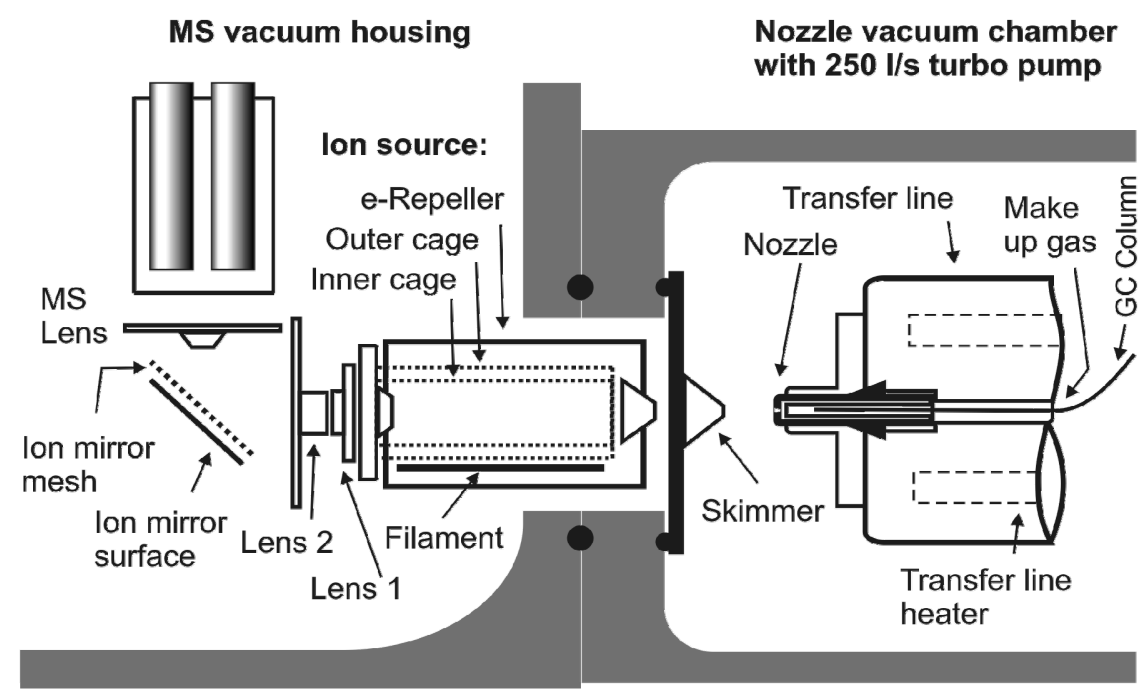

Figure 1. A schematic diagram of the 5975-SMB GC-MS with Cold EI. It is based on the Aviv Analytical conversion of Agilent 7890 GC + 5975 MSD (or 5977 MSD) into GC-MS with Cold EI. The various elements are indicated by their names.

molecular ion since it is fairly abundant and there is nothing in the mass spectrum above it. In addition, the use of low electron energy and or cluster CI (available via a method change) can further serve to confirm the molecular ion identity.

3) Improved library search and identification capabilities.

One of the most important advantages of GC-MS and its standard EI ion source is that EI mass spectra of about 600.000 compounds are available in extensive libraries that enable easy to obtain, fast and automated identification. Cold EI mass spectra, with their enhanced molecular ions, retain the lower mass fragments and are thus fully compatible with library based sample identification. Furthermore, while the enhancement of the molecular ions reduces the library (such as NIST) matching factors it often improves the library identification probability factors since the matching to competing candidates is largely reduced via the enhancement of the molecular ion, as described by Amirav, and Alon (2012) [3]. Additionally, the availability of the molecular ions provides a good "manual" confirmation (or rejection) of the library identification via the identity of the molecular ion and enables its further automated confirmation or rejection via isotope abundance analysis, such as with the TAMI software (Alon, and Amirav, 2006 and also the Aviv Analytical Website-TAMI page) [4,5], as described below.

4) Provision of accurate isotope distributions of the molecular ions.

Cold EI provides abundant molecular ions with accurate isotope distributions without any ion source related self chemical ionization or vacuum background interference, and thereby with unperturbed isotope distributions. Isotope Abundance Analysis (IAA) provides important information by itself (as used with isotope ratio MS and for isotope labeling experiments) and enables the elucidation of elemental formula of an analyte (Alon, \& Amirav, 2006) [4]. The Tal-Aviv Molecule Identifier (TAMI) software [5] was developed in order to provide a new method for identification, and even though it works well with standard EI sources, it truly excels when a Cold EI source is used. TAMI automatically converts the molecular ion group of isotopomers into elemental formulas and automatically (zero clicks) confirms or rejects the identification results of the NIST library. As a result, Cold EI with TAMI provides the ideal and ultimate sample identification technology for low cost unit resolution quadrupole MS.

5) Provision of elemental formulas with unit resolution quadrupole MS.

Clearly, it is highly desirable to be able to obtain elemental formula from unit resolution quadrupole based GC-MS systems. Along with the isotopomeric information of the molecular ion which enables IAA as described above, an identification procedure can and should consider the exact mass of the molecular ion, even though a quadrupole based \pm 0.1 amu mass accuracy is used (an extended explanation is provided by Alon, and Amirav, 2013 [6]). GC-MS with Cold EI is superior to GC-MS with high resolution TOF in the provision of elemental formula since without trustworthy molecular ions HR-TOF can not provide elemental formula, while Cold EI provides it for extended range of compounds that are amenable for analysis (The topic of extended range is discussed in B below). The combination of library identification which provides sample compound name and isomeric structure with the TAMI software which provides automated library confirmation or rejection as well as sample elemental formula (based on both IAA and quadrupole based medium accuracy masses) plus the 
Cold EI interface which provides those much needed enhanced molecular ions, is superior to LC-MS based sample identification as further discussed by Amirav (2012) [7].

6) Increased isomer and structural MS information.

Standard EI mass spectra excel in the provision of informative structurally related fragments (unlike with CI or ESI) that can serve for structure elucidation as discussed in a few books. Cold EI further enhances high mass fragments MS peaks and as a result provides even more structural and isomer MS information as shown in the analysis of drugs with the same elemental formula but different structures (Amirav, 2012) [7] and in the analysis of hydrocarbon isomers (Amirav, 2012) [8]. Clearly Cold EI is much closer to the ideal ion source in the provision of abundant and informative high mass spectral fragment peaks as these MS peaks can be related to the molecular ion which is often absent in standard EI.

7) Fragmentation Tunability.

Tunable fragmentation enables the elucidation of order of fragmentation hence helps to obtain the molecular structure. In Cold EI in view of the elimination of intraion thermal energy (which is controllable by itself, see point 8 below), the electron energy is the only remaining parameter that controls the intra ion energy, and the reduction of the electron energy allows better control and reduction of the degree of molecular ion fragmentation. While Cold EI does not provide full fragmentation control (in many cases the effect is small), it still clearly improves on this feature in comparison with standard EI.

8) Classical EI mass spectra with the Fly-through ion source.

While Cold EI outperforms standard EI, some users feel uncomfortable without the ability to obtain "classical EI” mass spectra. Such mass spectra can be beneficial if the need arises to distinguish between certain isomers via the library. Cold EI, unlike CI, enables method based (software controlled) easy and fast switching to "standard" EI for the provision of classical EI mass spectra. This conversion is simply performed via lowering the Cold EI make up gas flow rate to $5 \mathrm{ml} / \mathrm{min}$ without any need to physically replace an ion source or open the vacuum chamber (Gordin, Amirav, and Fialkov, 2008) [9]. Furthermore, one can use slightly higher flow rate and obtain slight enhancement of the molecular ions which results in having the best matching factors with the NIST library since the NIST search algorithm emphasis the molecular ions (Gordin et al., 2008) [9].

9) Cluster chemical ionization (CCI).

Cluster CI mode of ionization is provided with the fly-through ion source via a simple method based addition of methanol vapor from a methanol vial into the Helium make up gas (Fialkov, \& Amirav, 2003 and also Dagan, \& Amirav, 1996) [10,11]. Cluster CI further en- hances the molecular ions and results in having protonated molecular ions and methanol satellite $(\mathrm{M}+32$ and/or $M+33$ ) ions that serve to ensure the identification of the molecular ions masses. This is possible as fragments do not show such satellite peaks since the cluster is the first to fragment. Cluster CI was found by us as useful especially for the full confirmation of molecular ions in the monitoring of synthetic organic chemical reactions.

10) Deuterium Exchange

Cold EI enables a unique mode of intra-nozzle deuterium exchange that helps in $\mathrm{OH}, \mathrm{NH}$ and $\mathrm{SH}$ groups identification for further improved structural and isomeric elucidation (Dagan et al. 1996) [11]. While deuterium exchange gains popularity in LC-MS due to its provision of valuable structural information, it is not used in standard GC-MS. Probably this is since in standard GC-MS costly deuterated solvents should be used while the sample can re-exchange hydrogen in the column and ion source. With Cold EI a small amount of deuterated methanol or heavy water is used for many samples and back exchange can not occur in the column or at the ion source since the deuterated solvent is added after the sample elution and the ion source is fly-through type.

11) Isomer Distribution Analysis

As often the case, new instrument concepts leads to the development of new and powerful methods of analysis. The enhancement of the molecular ions obtained with Cold EI and the unique availability of these important ions for branched hydrocarbons and other types of isomers led to the development of isomer distribution (abundance) analysis. This type of analysis is an important new method for fuel and hydrocarbon mixture characterization via their origin unique isomer distribution pattern. This important new method is discussed by Fialkov, Gordin, and Amirav (2008) [12] and several applications are presented online (Advanced GC-MS Blog Journal) [13].

\subsection{Extending the Range of Compounds Amenable for GC-MS Analysis}

It is well recognized and known that the Achilles heel of GC-MS is its limited range of volatile and thermally stable compounds that are amenable for analysis. This limitation emerges from GC injector, column and ion source induced sample degradation and/or lack of sufficient vaporization. The limited range of compounds amenable for GC-MS analysis is further exacerbated by the fact that the relative abundance of the molecular ion is reduced with the sample compound size (mass) and for large compounds even if they elute, without trustworthy molecular ions there can not be properly analyzed. In CI the ion source limitation is even greater than in EI in view of its closed structure. GC-MS with Cold EI en- 
ables significant extension of the range of compounds amenable for analysis via the use of short columns with high column flow rates in combination with full elimination of intra-ion-source degradation and exhibition of abundant molecular ions. GC-MS with Cold EI provides the ultimate range of compounds amenable for GC-MS analysis and effectively bridges the gap between standard GC-MS and LC-MS. The key parameter for this unique capability is the use of very high column flow rates which in combination with the use of shorter columns leads to significantly (up to $200^{\circ} \mathrm{C}$ ) lower elution temperatures, while the provision of the enhanced molecular ions compensates for the traded GC resolution. High column flow rate further reduces intra injector liner degradation (lower elution temperature from the liner to the column and reduced liner residence time) and intraion-source sample dissociation is inherently avoided due to the Cold EI fly-through ion source geometry. Fialkov, Gordin, and Amirav (2003) [14] provide more details of this important feature and our perception of the range extension is further illuminated in Figure 2 and its caption. Improved GC-MS range of compounds amenable for analysis can be separated into three groups as below:

1) Significantly extended range of thermally labile compounds that are amenable for analysis.

The significantly lower elution temperatures from the GC column in combination with the use of temperature programmable injector with high injection flow rates enables a major increase in the range of thermally labile compounds that can be analyzed which is complemented and supplemented by the Cold EI enhanced molecular ions that proves the elution of intact thermally labile sample compound. In fact, GC-MS with Cold EI is equivalent and even superior to LC-MS with APCI or APPI in its range of thermally labile compounds that are amenable for analysis. In APCI and/or APPI the sample is

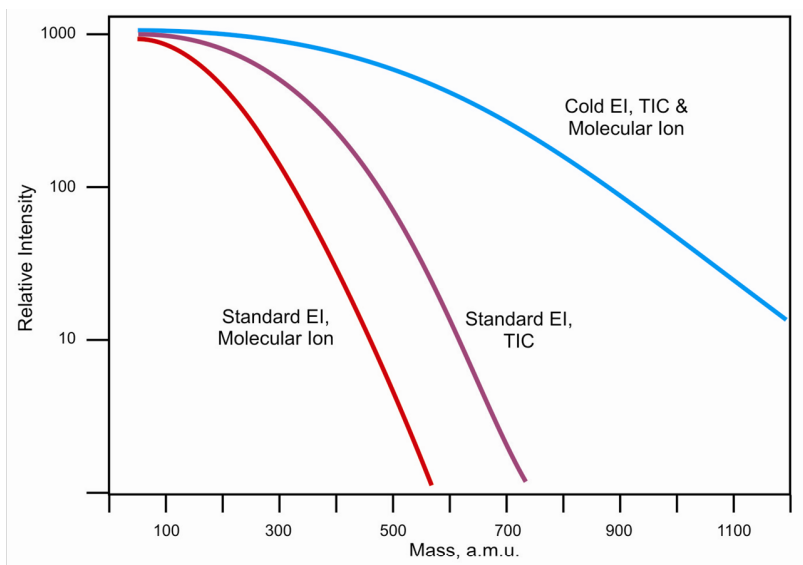

Figure 2. A cartoon that demonstrates our perception of the average sensitivity gain of Cold EI with the 5975-SMB versus sample mass, which at some point is transformed into extended range of compounds amenable for analysis. thermally vaporized at a very hot vaporization (liner like) oven $\left(400^{\circ} \mathrm{C}-500^{\circ} \mathrm{C}\right)$ where the sample stays for a short time while in GC-MS with Cold EI the sample is vaporized at much lower injector temperatures and spends much longer time at the column at much lower temperatures. Since the effect of thermal degradation exponentially depends on the temperature, Cold EI can be gentler to thermally labile compounds than APCI/ APPI.

2) Significantly extended range of low volatility compounds that are amenable for analysis.

The significantly lower elution temperatures from the GC column with high short column flow rates enables a major increase in the range of low volatility compounds that can be analyzed. It was found that it is possible to approximately double the molecular weight and size limit of compounds that can be analyzed (in comparison with standard GC-MS) to about 1200 amu for non polar compounds and 800 amu for polar compounds. In fact, GC-MS with Cold EI is equivalent in its range of compounds to LC-MS with APCI or APPI.

3) Significantly extended range of polar compounds that are amenable for analysis.

The elimination of ion source tailing and degradation on its metallic surfaces enables the analysis of many polar compounds without their derivatization. One example is given in the work of Amirav, Seltzer and Hefetz (2013) on the analysis of free fatty acids in ant heads [15].

\subsection{Speed-Faster GC-MS Analysis}

The reduction of analysis time is an obvious needed GC-MS improvement. While there are few ways to reduce the analysis time they are all involved with tradeoffs and thus fast GC-MS is an art of finding the optimal trade-off. GC-MS with Cold EI enables the highest capability fast GC-MS, from the reduction or elimination of sample preparation to the final fast analysis results. Basically, fast GC-MS is achieved based on the trade-off of GC resolution for speed of analysis and compensation for the sacrificed GC separation with enhanced separation (selectivity) power of the MS and/or MS-MS via the enhancement of the molecular ion. A few GC-MS aspects can be improved for achieving faster analysis as discussed below:

1) Faster splitless injection

In standard GC-MS the splitless injection takes a minute plus another minute or more for the temperature of the GC oven to rise and start the separation process, plus another minute or more for cooling down from that initial separation temperature to about $50^{\circ} \mathrm{C}$ that is needed for the splitless injection cryo-focusing. While split injection can be much faster via avoiding the above wasted time problems, it often involves with unacceptable signal loss. GC-MS with Cold EI uniquely enables simple syringe based fast splitless injections. With Cold EI, very high 
injection column flow rates can be used such as 30 - 60 $\mathrm{ml} / \mathrm{min}$ hence the splitless injection takes a second or two, similarly to a split injection, and it can be performed at higher initial GC oven temperature, thereby saving a few precious minutes from the injection time, temperature program rise and cooling down time.

2) Freedom in choice of the most suitable column.

Standard GC-MS typically uses $30 \mathrm{~m}$ columns with $0.25 \mathrm{~mm}$ ID and $1 \mathrm{ml} / \mathrm{min}$ column flow rate which restricts the analysis time. The use of a shorter $0.1 \mathrm{~mm}$ ID microbore column can shorten the analysis time by a factor of 2.5 while retaining the separation but with a trade-off of on-column sample amount, column loadability (sample capacity) and column lifetime. Fast GC-MS with Cold EI is characterized by an unrestricted selection of column type, ID, length and flow rate and in this aspect GC-MS with Cold EI is similar to LC-MS in which the user can select any column length, diameter and particle size for optimal trade-off of separation with speed of analysis. Note that with the reduction of the column length and/or increase in its flow rate, every reduction by a factor of two of the GC separation peak capacity enables four times faster analysis. Furthermore, while the use of short columns facilitates faster analysis, the use of high column flow rates is preferable since it can be controlled without any hardware change and time programmed with sub second response time (unlike GC oven temperature). As a result, GC-MS with Cold EI enables the optimization of the GC-MS analysis method for achieving the shortest analysis time while delivering the needed results via an optimized selection of column ID, length and flow rate.

3) Ultra-fast ion source response time.

While the fast GC-MS literature is full of discussions conducted by proponents of time of flight MS about the need for fast scan speed, no one mentions the equally or even more important role of fast ion source response time and elimination of ion source peak tailing. In the analysis of semi-volatile and/or polar compounds GC-MS peak tailing originates in the ion source due to its slow response time and hampers the GC separation particularly in fast GC-MS and/or GCxGC-MS. Cold EI on the other hand is characterized by ultra fast sub millisecond ion source response time and full elimination of any ion source tailing. A full discussion of this aspect is provided by Amirav, Alon, and Fialkov (2013) [16].

4) Compatibility with the scanning speed of quadrupole MS.

Quadrupole is the most widely used mass analyzer in GC-MS. However, it has a somewhat limited scan speed which today is in the $10,000-20,000 \mathrm{amu} / \mathrm{s}$ range by most vendors. The use of fast GC-MS through the use of high flow rate and short widebore $(0.53 \mathrm{~mm}$ ID) or standard narrowbore $(0.25 \mathrm{~mm}$ ID) columns provides fast analysis with peak width in the order of $0.5 \mathrm{~s}$ (or more) that are fully compatible with the scan speed of quadrupole MS that can exceed $20 \mathrm{~Hz}$ scan rate for $50-550$ amu mass scan range.

5) Extract-free dirty sample introduction without lengthy sample preparation.

While the chromatographic separation could be fast, for truly fast analysis the time devoted for sample handling and preparation must be reduced as well. The use of the ChromatoProbe for sample introduction for its intra GC injector thermal desorption facilitates extractfree sample introduction for broad range of solid and sludge samples and thereby reduces the time devoted for sample handling and clean up. The use of the ChromatoProbe with GC-MS with Cold EI benefits from a higher possible injector flow rate for easier and softer sample thermal desorption with higher recoveries. More information on the ChromatoProbe is provide by Amirav, and Dagan (1997) [17] and in the product website [18]. In addition, using short columns and high column flow rates combined with flow programming at the end of the analysis guarantees that every compound that is introduced from the injector into the column is eluted. Consequently, faster and simpler forms of sample preparation such as immersion and injection can be practiced as described in the analysis of synthetic cannabis performed by Amirav (2012) [19].

6) Improved compatibility with low thermal mass ultra fast GC.

When the sample contains several compounds with broad volatility range, fast analysis requires using a low thermal mass (LTM) fast GC that can provide fast temperature programming rate. An LTM Fast GC was developed, which enables full analysis cycle time of under one minute with temperature programming rate of up to $2000^{\circ} \mathrm{C} / \mathrm{min}$ and cooling back time of under 10 seconds (Fialkov, Morag, and Amirav, 2011) [20]. GC-MS with Cold EI is fully compatible with the LTM Fast GC use of short columns and high column flow rates (Fialkov et al., 2011) [20]. High column flow rates are highly desirable for fast temperature programmed fast GC-MS in order to elute the late eluting compounds before the high temperature plateau end of analysis where the peaks can be broadened and delicate samples can decompose.

7) Shorter sample handling time - Open Probe Supersonic Fast GC-MS.

The recently developed Open Probe Fast GC-MS is the latest and most advanced development in the field of real time analysis. In addition to real time analysis, and unlike any other real time analysis technique, it also provides separation and library identification. The Open Probe (Poliak, Gordin, and Amirav, 2010) [21] further reduces the time devoted for sample collection and preparation. Amirav, and Alon (2013) [22] provide fur- 
ther information on the Open Probe Fast GC-MS including its comparison with DART and DESI.

\subsection{Sensitivity}

GC-MS sensitivity is its prime specification and although not very important for qualitative applications it serves for many users as a symbol of the system quality. However, the OFN specification that is used to measure GCMS sensitivity is inappropriately provided by all GC-MS vendors and does not properly represents the GC-MS sensitivity (or LOD) as discussed by Fialkov, Steiner, Lehotay, and Amirav (2007) [23] and further discussed by Amirav (2012) [24]. The subject of GC-MS sensitivity is more involved than commonly perceived and GCMS sensitivity depends on several parameters and performance features that can be improved as explained by Fialkov et al. (2007) [23], by Amirav et al. (2008) [1] and in the points listed below. As discussed below, Cold EI improves the GC-MS sensitivity (lowers its LOD), the 5975-SMB GC-MS with Cold EI is the most sensitive GC-MS and the harder the compound for analysis the greater is the Cold EI gain in sensitivity.

1) Ion source signal strength.

Standard EI ion source is operated with 30-100 $\mu \mathrm{A}$ emission current and with few spiral paths of electron motion that are magnetically confined. This ion source provides good ionization efficiency which nowadays approaches $0.1 \%$ at the ion detector ( $\sim 600$ OFN molecular ions per femtogram in SIM mode). The Cold EI flythrough ion source is operated with 8 - $12 \mathrm{~mA}$ emission current and with about three cycles for each ionizing electron thus it is more sensitive than standard EI despite the $\times 10$ faster motion velocity of sample compounds in the SMB. However, SMB skimmer collimation losses $(\times 4-5)$ and ion mirror losses $(\times 3)$ reduce the Cold EI overall ion signal which is similar to that of standard EI. The TIC signal of Cold EI is slightly (up to $\times 1.5$ ) lower than that of the Agilent 5975 EI ion source for small molecules and higher (without limitations) for large compounds. Naturally, these numbers are time dependent as improvements are steadily introduced and Cold EI as a younger technology has a lot of potential for future improvements.

2) Ion source noise - The elimination of vacuum background noise.

Standard EI ion sources have two major noise sources, vacuum background and helium metastable (neutral) related mass independent noise. While the mass independent noise was recently suppressed in standard EI ion sources via the use of improved ion detector and ion optics designs (such as the Agilent triple axis ion detector), vacuum background still remains and is the most prominent source of noise. While OFN specifications are obtained in a mass spectral region that has a minimal vac- uum background noise, with a new and clean vacuum system and with electro-polished ion source surfaces (that are scratched and become rough after the first ion source cleaning), in real world applications vacuum background could be high and severely limit the obtained $\mathrm{S} / \mathrm{N}$. In Cold EI, vacuum background is filtered out in the dual-cage fly-through ion source. This filtration is enabled due to the fact that sample compounds in the supersonic molecular beam have a few eV directional kinetic energy, while vacuum background species have non-directional $<0.1 \mathrm{eV}$ kinetic energy at the ion source. Thus, the combination of directional SMB compound motion and a small electrostatic repulsion potential in one of the fly-through ion source lenses fully eliminates vacuum background noise. This improved reduction of noise level improves the obtained S/N in Cold EI which are superior to any standard EI ion source and can lead to noise free OFN mass chromatograms which allows OFN $1 \mathrm{pg}$ RSIM S/N specification of any desirable value including $>10^{+6}$.

3) Elimination of mass independent noise.

Helium related mass independent noise is a one type of noise that exists in all GC-MS systems. It emerges from the co-formation of metastable helium atoms during the electron ionization process, and these metastable atoms can either directly ionize molecules upon collisions or generate free electrons upon there scattering from surfaces that can directly contribute to noise or ionize sample and/or vacuum background compounds after the mass analyzer. Recently, curved pre quadruple ion optics and improved ion detectors with multiple axis ion paths significantly reduced this type of mass independent noise. However, the effect of such mass independent noise reduction on the overall sensitivity is limited since the most important type of noise caused by vacuum background still remained. In Cold EI vacuum background is fully eliminated as above, making the elimination of mass independent noise a relatively more important task. In the 5975-SMB GC-MS with Cold EI a $90^{\circ}$ ion mirror is used in order to obtain further significant suppression of mass independent noise, and as a result its mass independent noise count rate is very low, below 5 ions/s.

4) Reduced column bleed and ghost peaks noise.

Column bleed with its multiple mass peaks of $\mathrm{m} / \mathrm{z}=$ 73, 147, 207, 281, 355, 429, 503 etc. is a known major source of noise that hampers the detection and identification of low volatility compounds. In addition, ghost peaks which belong to previous runs (sample compounds that elute in the next few runs) further complicate and increase the apparent column bleed noise. Cold EI enables the use of shorter columns with higher column flow rates and column flow programming at the end of the run. As a result, the sample compounds elute at significantly lower temperatures (Fialkov et al., 2003) [14] so that 
column bleed and ghost peaks noise can be eliminated altogether. The lower elution temperatures and flow programming column cleaning significantly reduce any ghost peak. In addition, the use of transfer-line temperature programming further reduces PDMS related bleed noise to a minimum and thus Cold EI is characterized by exceptionally low noise.

5) Enhanced molecular ions for improved sensitivity.

The molecular ion is by far the most informative and selective ion. Thus, for the sensitive and selective detection of any compound it is advised to monitor it via its molecular ion either in SIM or full scan RSIM modes. Thus, the enhancement of the molecular ion directly improves the sensitivity (lower LOD). In Cold EI the molecular ion is enhanced while keeping the total ion count. The degree of enhancement can be small or modest for small and rigid compounds such as benzene or OFN but large, more than three orders of magnitude, for large aliphatic compounds as demonstrated and discussed by Fialkov et al. (2007) [23], by Amirav et al. (2008) [1] and by Amirav et al. (2012) [2]. As a result, Cold EI particularly excels in the sensitive detection of large and difficult to analyze compounds.

6) Reduced ion source peak tailing.

One of the adverse GC-MS EI ion source effects that limits its sensitivity is ion source peak tailing. A small chromatographic peak tail hides, like an iceberg, a significant loss of TIC signal. Ion source peak tailing can be reduced at increased EI ion source temperature but as the ion source temperature is increased the molecular ions are exponentially reduced for many classes of compounds and intra-ion-source degradation is promoted. Ion source peak tailing reduces the chromatographic separation, increases the signal RSD, lowers the sample signal and increases its MS noise. With Cold EI, ion source related peak tailing is fully eliminated in view of the use of a contact-free fly-through ion source. Thus, as the sample size and/or polarity are increased and its volatility is reduced the gain in $\mathrm{S} / \mathrm{N}$ with cold EI is significantly increased. The tailing-free ultra fast ion source response time is provided with Cold EI, regardless of the sample's volatility. Peak tailing, its consequences and the way to eliminate it, are discussed by Amirav et al. (2013) [16].

7) Improved ion source inertness for increased range of thermally labile compounds that are amenable for analysis.

Regardless of the selection of ion source materials and the various claims made by vendors, standard EI ion sources are active due to unavoidable contact of sample compounds with metal surfaces, and since all metals act as catalysts to degrade many types of organic compounds. The use of electrically conductive materials such as metals is essential at the ion source to create optimal electric fields in it. As a result, standard EI ion sources are not inert and induce sample decomposition for many compounds. Cold EI is an inherently inert ion source since it uses a contact-free fly-through ion source configuration. No sample contact inherently means no sample degradation at the ion source. This feature of ultimate ion source inertness leads to enhanced sensitivity particularly when it is most needed in the analysis of sample compounds that are difficult to analyze such as thermally labile compounds.

8) Improved compatibility with large volume injections.

Large volume injection (LVI) is a known technique that can improve the concentration sensitivity and provide lower detected concentration. It is usually performed via the injection of larger than the standard $1 \mu \mathrm{L}$ sample volume with a temperature programmable GC injector. However, at some point the injection of further larger volume leads to increased column, liner and ion source contamination while the increased signal is offset by similarly increased matrix noise. With Cold EI the matrix interference on the molecular ion is minimal (Kochman, Gordin, Goldshlag, Lehotay, and Amirav, 2002) [25] thus Cold EI can further benefit from LVI. Furthermore, with the use of short column and flow programming at the end of the run, practically everything that entered from the liner into the column elutes, thereby keeping the column clean. Cold EI enables another convenient mode of "Larger Volume Injection” that utilizes standard splitless injections with very high pulsed splitless flow rates that are possible with short columns (such as $30-60 \mathrm{ml} / \mathrm{min}$ ). In such injections conditions, the sample is vaporized and swept into the column at a rate of $>1 \mu \mathrm{L}$ per second hence injection volume in the range of 2 - $10 \mu \mathrm{L}$ can be employed with standard injectors without injector temperature programming and its related loss of volatile sample compounds.

9) Reduced matrix interferences.

Matrix interference is the most important source of noise that limits the LOD in the analysis of samples in complex matrices such as drugs in urine or blood and/or pesticides in agricultural products. However, it was found that matrix interference is exponentially reduced with mass by a factor of 20 every 100 amu (Kochman et al., 2002) [25] as also discussed in the work of Amirav (2013) [26]. Thus, the enhancement of the molecular ions in Cold EI enables the detection of the sample compounds with significantly less matrix interference hence with much lower LOD. In fact, the detection of pesticides in agricultural products via the molecular ions in Cold $E I$ is as selective and sensitive as their detection by MS-MS on a fragment parent ion in standard EI. When MS-MS is employed on the molecular ion the selectivity is further increased and both first and third quad resolution can be opened for higher signal and sensitivity. 
10) Lower level impurities analysis.

Another type of "sensitivity" which is not often discussed regards low level impurities analysis in a given material and the most known such requirement is in the analysis of impurities in active pharmaceutical ingredients (API) or in simple terms the analysis of impurities in drugs. This topic is separately discussed with examples in the work of Amirav (2013) [27]. While initially such analysis seems simple as the required detection limits are typically $0.1 \%$, in fact it is a challenging application. The challenge begins with the fact that uniform response is needed in order to know the concentration of the impurity without knowing its identity and without requiring a lengthy procedure of impurity sample calibration. Two factors are most detrimental for this type of analysis; a) Drugs are typically polar compounds and so are most of their impurities and even if they are thermally stable they tend to saturate the column at $<10 \mathrm{ng}$ amount via the formation of peak fronting. When the GC peak starts to exhibit fronting it means that any additional on-column sample amount will not increase the peak height but it will only broaden the peak via its increased front hence hamper the analysis of nearly co-eluting impurities. It was found that most drug impurities are either isomers or homologous and related compounds that elute near the main API compound in GC-MS. b) Ion source related peak tailing reduces the total ion count signal for polar drug like compounds. When the column capacity is below $10 \mathrm{ng}$ and the TIC signal to noise ratio is below 100 (peak to peak) for $1 \mathrm{ng}$ impurities, at the $0.1 \%$ concentration level they are barely or not detected and certainly can not be identifies. With Cold EI the peak tailing losses are eliminated thus it provides better TIC sensitivity. In addition, the use of high column flow rates increases the column capacity which depends on the separation plate film volume that linearly increases with the flow rate. As a result, Cold EI excels in the analysis of impurities and can detect $0.1 \%$ impurities with $\mathrm{S} / \mathrm{N}>100$ (peak to peak noise) [27]. Recently, the need for genotoxic impurities analysis is growing and in such analysis much lower concentration detection limits are required.

11) Reduced limit of identification.

Often detection must include trustworthy identification. While the subject of what is meant by identification is not trivial it is easy to agree that improved identification relates to improved total ion count signal to noise ratio plus having more abundant sample compound characteristic ions such as the molecular ions and high mass fragment ions. Cold EI enhances the molecular ions and high mass fragments and provides improved total ion count signal to noise ratio that can be $>1000 /$ ng.

12) More representative sensitivity specifications.

Clearly, the current use of OFN full scan RSIM specification is inappropriate and misleading (Amirav, 2012)
[24]. GC-MS sensitivity should be characterized by several specifications given for a range of compounds that include OFN (as the easiest to analyze compound) plus a few other compounds that are gradually more difficult to analyze. The test mixture of the Aviv Analytical 5975SMB GC-MS with Cold EI includes OFN, Hexadecane (n- $\left.\mathrm{C}_{16} \mathrm{H}_{34}\right)$, Methylstearate, Cholesterol and $n-\mathrm{C}_{32} \mathrm{H}_{66}$. Representative specifications can include: a) RSIM S/N on all the mixture compounds; b) SIM S/N; c) OFN signal in number of molecular ions per femtogram; d) Total ion count (TIC) $\mathrm{S} / \mathrm{N}$ for all the test mixture compounds, which in our opinion is the most representative sensitivity specification as it includes both signal and square root of the background noise level; e) TIC over baseline ratios which relate to the identification limits.

\subsection{Uniform, Compound Independent Ion Source Response}

Uniform compound independent response is a highly needed feature which is absent in LC-UV and/or LC-MS. GC with FID is well known to be a semi-quantitative analytical tool while GC-MS is similar to GC-FID for volatile sample compounds but its response uniformity is eroded for semi volatile compounds due to ion source related peak tailing. Uniform response provides the ability to know the relative amount of any unknown compound or impurity without its separation, identification, synthesis and the performance of compound specific calibration curves. Thus, uniform response is of particular importance in the areas of drug impurities analysis and for the elucidation of chemical reaction yields as discussed in the work of Amirav on drug impurities analysis (2013) [27] in the work of Amirav, Gordin, Belgorodsky, Seeman, Gozin and Fialkov (2013) on the measurement and optimization of organic chemical reaction yields [28] and in the work of Amirav, Gordin, Hagooly, Rozen, Belgorodsky, Seemann, Marom, Gozin, and Fialkov, (2012) [29].

1) Uniform, compound independent ion source response.

The electron ionization cross section approximately depends on the number of electrons in the sample compounds hence on its molecular weight thus sample weight. As a result, for volatile compounds the EI TIC mass chromatograms provide uniform compound-independent peak area responses, similar to those of GC-FID. However, as the sample compound becomes bigger, more polar and/or less volatile, ion source peak tailing becomes more and more pronounced and consequently the standard EI ion source response uniformity is eroded and lost. Cold EI provides uniform response regardless the sample volatility and provide it for about doubled range of compounds amenable for analysis. This feature is translated into a unique Cold EI capability-the provi- 
sion of chemical reaction yields, something that is missing in ESI LC-MS or standard GC-MS systems.

\subsection{Improved GC-MS Compatibility with Enhancement Technologies}

Enhancement technologies are important add-on techniques, devices and software that serve to further improve the performance of GC-MS. Usually they are offered as options to the basic GC-MS systems. Known enhancements are the CI ion source, MS Probe, bigger Turbo molecular pump, MS libraries, auto-samplers and thermal desorption units. Clearly, improved compatibility with enhancement technologies can further improve GCMS as described below.

1) MS Probe, ChromatoProbe and Intra Injector Thermal Desorption Devices.

GC-MS can benefit from having MS probe. One type of such MS probe is the ChromatoProbe sample introduction device that provides fast probe sampling and instant ChromatoProbe/GC-MS switching (Amirav et al., 1997) [17]. The ChromatoProbe is based on the conversion of a GC injector into an MS probe that accepts samples in micro-vials when the injector is connected to the ion source via a short $1 \mathrm{~m}$ micro bore transfer-line. The ChromatoProbe also uniquely enables the injection of very "dirty" samples without any sample preparation (Amirav et al., 1997) [17] when it serves as a thermal desorption unit behind a standard analytical column. The use of high flow rate with short transfer-line capillary enables significantly extended range of compounds amenable for analysis with Cold EI and combined with the fully inert Cold EI ion source and the provision of enhanced molecular ions the ChromatoProbe operation as an MS probe excels and is more effective and informative with Cold EI than a standard Probe is with a standard EI ion source. Similarly, intra injector thermal desorption is more effective and can be performed at lower injector temperatures when operated with higher column flow rates that are possible with Cold EI.

2) Tal Aviv Molecule Identifier Software (TAMI).

The TAMI [5] provides automatic confirmation or rejection of NIST library search results and in case of a rejection it performs an independent search for the analyte's elemental formula, providing a table of possible elemental formula with a declining probability of matching to the experimental isotope abundances and molecular ion measured mass even with unit resolution single quad GC-MS. TAMI requires the provision of accurate isotope abundances and thus, for proper compatibility with it, the sample must exhibit abundant molecular ions free from vacuum background and/or residual ion source self chemical ionization. Cold EI, unlike standard EI, excels in the provision of such analysis conditions, and provides trustworthy data for extended range of com- pounds. Thus, a quadrupole based GC-MS system, coupled with Cold EI, is more effective in the provision of elemental formulae than expensive GC-MS with high resolution TOF and standard EI.

3) Pulsed Flow Modulation GCxGC-MS.

An effective type of GCxGC modulation method named pulsed flow modulation (PFM) was developed for its combination with GC-MS (Poliak, Kochman, and Amirav, 2008) [30] and also Poliak, Fialkov, and Amirav, 2008) [31]. It is a simple and low cost type of GCxGC modulation method and device that does not require any cryogenic gas or liquid. However, it requires compatibility with second GCxGC column flow rates of $\sim 20$ $\mathrm{ml} / \mathrm{min}$ which is not a problem with FID, FPD or other types of GC detectors but is incompatible with standard GC-MS flow acceptance that requires significant flow splitting. However, Cold EI is seamlessly compatible with the pulsed flow modulation GCxGC flow rate requirements, providing the ultimate in both sensitivity and sample information.

4) Low Thermal Mass Fast GC for ultra fast GC-MS.

Currently, a few low thermal mass fast GC systems are available. The most widely used is of Agilent (previously RVM). Recently, a unique LTM Fast GC was developed with full analysis cycle times of under one minute (50 $350-50^{\circ} \mathrm{C}$ ) with temperature programming rates of up to $2000^{\circ} \mathrm{C} / \mathrm{min}$ and cooling back time of under 10 seconds (Fialkov et al. 2011) [20]. This "Supersonic Fast GC" (possible future name) is fully compatible with the use of any fused silica short column and high column flow rate (Fialkov et al. 2011) [20]. However, one other feature that needs to be improved in fast GC-MS is the ability to perform fast splittless injections which require high column flow rate. Furthermore, fast GC-MS analysis of compounds in complex mixture requires the ability to periodically trim the column due to matrix contamination buildup at the front segment of the column near the liner, and to be able to replace the column at low cost. All the above mentioned improvements are uniquely met with the combination of the LTM Supersonic Fast GC and GC-MS with Cold EI (Fialkov et al. 2011) [20].

5) Open Probe Fast GC-MS.

The ultimate goal in fast analysis is to combine fast separation with fast or no sample preparation in order to approach the ultimate goal of real time analysis with separation. Recently, a few types of real time analysis techniques such as DART and DESI gained popularity, but these methods suffer from several deficiencies and do not provide separation and/or library based easy and trustworthy identification. As described in previous work by Amirav et al. (2013) [22] and Poliak et al. (2010) [21], the unique low thermal mass fast GC-MS was combined with Cold EI and with a novel Open Probe inlet for achieving fast sampling without sample preparation. The 
Open Probe is a probe-oven that is mounted onto the fast GC and which is open to room air with helium purge flow protection to eliminate air leakage. Thus, sample handling and introduction is as simple and fast as touching the sample (with a swab or melting point glass tube) and pushing it into the open probe. The Open Probe Fast GC-MS provides direct analysis in real time (DART) but in comparison with other types of DART it uniquely provides the following features:

a) Fast chromatography separation for improved mixtures analysis.

b) Library based sample identification is enabled combined with isotope abundance analysis software for best identification.

c) Cold EI uniquely provides uniform compound independent response for improved quantitation. Furthermore, quantitation by Cold EI does suffer as ESI or APCI from any ion suppression effects.

d) Extended range of thermally labile and low volatility compounds are amenable for analysis.

e) Swabs can be used to bring samples from remote surfaces combined with full thermal desorption.

f) An in-vacuum ion source is used hence the instrument cost less and the broad install base of Agilent 5975/7 GC-MS can serve for the accommodation of Open Probe Fast GC-MS.

g) The same system can be operated with a second injector as GC-MS.

h) No solvent is used unlike with DESI while the helium gas consumption is about 50 times lower than in DART.

In short, Open Probe Fast GC-MS with Cold EI establishes the ideal goal of achieving real time analysis with separation and library based identification.

6) Electron Ionization LC-MS in GC-MS.

LC-MS can significantly benefit from having electron ionization as it provides automated library identification and extensive fragment information for improved sample identification. In addition, EI does not suffer from ion suppression effects that plague ESI or APCI and it uniquely exhibits uniform compound independent ionization yield (in contrast to ESI) for improved quantitation. Thus, bringing back EI to LC-MS is highly valuable if a reliable and robust EI interface can be developed. Furthermore, yet another highly desirable goal is to have both GC-MS and EI-LC-MS in a one system with easy method based switching between these two modes of operation. A novel EI-LC-MS approach was developed, based on interfacing LC and MS with supersonic molecular beams (SMB) and sample ionization with electrons as vibrationally cold compounds in the SMB (Cold EI) (Granot, and Amirav, 2005) [32]. The output of an LC was vaporized behind a supersonic nozzle at about 0.1 Bar to suppress cluster formation yet to obtain effi- cient vibrational cooling, and the sample compounds expended into the vacuum system. Sample vaporization is based on spray formation followed by fast, thermal vaporization of the sample compounds prior to their expansion from the supersonic nozzle. It was demonstrated that the stage of spray formation and vaporization can be performed in a modified GC-MS injector that is coupled to the nozzle via a short heated transfer-line. Consequently, in a GC-MS with Cold EI one injector can serve for GC-MS analysis and a modified second injector for EI-LC-MS (Amirav et al., 2008) [1].

7) Backflush.

Backflush is a known effective technique to maintain clean GC columns when used in the analysis of complex matrices. In backflush, at the end of the analysis, when the GC oven is at its highest temperature or after the elution of the last to elute sample compound of interest, the flow in the column is inverted via the provision of high pressure at the end (or middle) of the column and low pressure at the injector. As a result, heavy compounds with low volatility that coat the early portion of the column migrate back the short distance to the injector and are eliminated from the column. However, it was found that some of the backflush devices are active and in-time develop major peak tailing. Several backflush devices are based on a metal structure that it deactivated by Silcosteel (Restek) thin fused silica film. This film can deteriorate after several heating and cooling cycles due to large differences in the thermal expansion coefficients of fused silica and stainless steel. Thus, while in sales demonstrations backflush is very effective, after short usage some devices become faulty and induce major peak tailing. A unique ultimate inert backflush device was developed, utilizing a simple 1/16” Swagelok $\mathrm{T}$ union that includes in its straight path a glass tube with $1.2 \mathrm{~mm}$ OD and 0.7 $\mathrm{mm}$ ID. The column and transfer line ends are brought inside this glass tube to a distance of $\sim 1-2 \mathrm{~mm}$ while the third (middle) input serves to bring about $1-2 \mathrm{ml} / \mathrm{min}$ make-up helium gas from an EFC. This way, the added gas flow rate dynamically focuses the output of the column into the transfer-line and the sample compounds do not adsorb on any surface except the column and transfer-line hence tailing is eliminated. This novel concept of gas dynamic flow focusing into a column was described and demonstrated in our papers on pulsed flow modulation GCxGC (both papers by Poliak et al. 2008) [30, 31]. While this backflush device is inert it requires the addition of some flow rate which is not an issue with GC-MS with Cold EI but could be a downside in standard GC-MS.

8) Thermal Desorption and Purge and Trap.

While these known devices are effective their compatibility with GC-MS can be improved via the increase of splitless column flow rate acceptance as provided with 
GC-MS with Cold EI.

9) Improved GC-MS-MS Performance.

MS-MS is a powerful GC-MS enhancement technology which helps particularly in the reduction of matrix interference in the analysis of target sample compounds in complex matrices. The major use of GC-MS-MS is in pesticide analysis in agricultural products and drug analysis in biological fluids. Cold EI can improve GCMS-MS performance in a few important aspects including: A) Improved selectivity. Cold EI enhances the abundance of the molecular ion which is the most selective ion in the mass spectrum. Furthermore, when the molecular ion serves in MS-MS as the parent ion the daughter ion mass is typically higher than when a fragment is used as the parent ion. Consequently, the MS-MS selectivity is significantly improved by an estimated two orders of magnitude in the use of molecular ion instead of a fragment ion as the MS-MS parent ion. B) Improved instrument sensitivity. While MS-MS on the molecular ion further reduces matrix interference it also serves to increase the number of daughter ions signal hence the instrument sensitivity. This improved MS-MS sensitivity emerges in two ways of: 1) Molecular ions as parent MS-MS ions require lower CID voltage and they dissociate in the CID process into lower number of fragment ions which are better retained by the RF only Q2. The molecular ion is easier to dissociate than a stable fragment ion that was formed in the EI process since abundant fragments are abundant as they are typically stable fragments and thus are harder to break. The higher typical CID voltage used with fragments creates more energetic lower mass daughter ions that are harder to retain in Q2. 2) The increased selectivity of MS-MS on the molecular ion can be translated into up to an order of magnitude higher signal via the use of lower Q1 and Q3 resolution. C) Extended range of compounds amenable for GC-MS-MS analysis. GC-MS-MS is mostly used with groups of target compounds such as pesticides and drugs, which include significant portion of thermally labile compounds. As a result, GC-MS-MS suffers from growing competition with LC-MS-MS on those types of analyses. Cold EI enables the analysis of much greater range of those pesticides and drugs and can even serve for the analysis of pesticides that are difficult to analysis by both GC-MS-MS and LC-MS-MS such as captan, captafol and folpet. Furthermore, GC-MS-MS with Cold EI can uniquely serve for the confirmation of LC-MSMS labile samples.

\subsection{Improved GC-MS Flexibility, Ease of Use and Price}

While not often discussed, flexibility, ease of use and price are all GC-MS parameters that can be improved and this section describes several areas of their possible improvements.

1) Unlimited selection of column parameters.

In LC-MS unlike in GC-MS users can select broad range of columns with various lengths, diameters, solvent types and solvent flow rates while GC-MS is practically restricted to $30 \mathrm{~m}$ columns with $1 \mathrm{ml} / \mathrm{min}$ helium flow rate. In GC-MS with Cold EI, any column can be used without restrictions on its diameter, length and flow rate. This feature allows optimal trade off of GC resolution, speed, sensitivity and range of compounds amenable for analysis and it significantly simplifies analysis method development.

2) Number of columns that can be simultaneously connected.

In GC-MS with Cold EI two columns can be simultaneously connected with the nozzle transfer line from two different injectors. Even three columns can be simultaneously connected in GC-MS with Cold EI with an additional third injector such as the Optic of ATAS-GL. This feature improves the GC-MS flexibility in a few ways such as enabling fast screening with a short column followed by confirmation with a longer column. Similarly, it enables the use of one injector with the ChromatoProbe either as an MS probe or behind a separation column for intra injector thermal desorption of solids or sludge samples while a second injector can serve for standard syringe based injections. In addition, one injector and column can serve for GC-MS analysis while the second injector can be modified and serve for EI-LC-MS or flow injection analysis as described in 40 above. Another utilization of the possibility of using two columns that are connected together to the Cold EI nozzle is to have one injector devoted for standard GC-MS analysis while the second column can be connected with an Open Probe Fast GC-MS as briefly described in 39 above. In short, the ability of having two simultaneously connected columns with the nozzle and Cold EI ion source is highly desirable as it improves the system's flexibility.

3) Columns replacement and injector service without breaking vacuum.

One further aspect in which GC-MS can be improved is to make its service easier via enabling the replacement of its GC column and injector liner or septum without venting the MS vacuum chamber and/or full injector and transfer line cooling. For GC-MS with standard EI a few "no-vent" devices were developed which require the addition of another EFC. In GC-MS with Cold EI the column output is designed to be able to tolerate atmospheric pressure and the make up gas EFC is already available. Thus, during column replacement the nozzle flow rate is increased to form nozzle pressure of $1100 \mathrm{mBar}$ while the injector flow rate is off (1 Bar pressure). As a result, the column flow is reversed and thus liner or septa can be replaced while the column is protected from air penetra- 
tion. Similarly, when the transfer-line is open for column replacement helium flows out and purge protects the transfer line from the penetration of air although small amount of air flow is actually harmless to the fly-through ion source when its filament is off.

4) Column flow programming and reduced column flow rate effects on ion source response.

In standard EI the ion source is designed to maximize the sample ionization yield at about $1 \mathrm{ml} / \mathrm{min}$ helium flow rate. Above this flow rate the ion source response begins to decline, so that a new tune is required, and above a few $\mathrm{ml} / \mathrm{min}$ the ion source response begins to sharply reduce due to extended intra-ion space-charge effects. In contrast, Cold EI has no ion source flow rate effects. In Cold EI the nozzle back pressure is stabilized at values of about 1 Bar (usually at $700 \mathrm{mBar}$ ) and as the column flow rate is modified, the added helium make up gas flow rate is automatically changed to maintain and stabilize the set nozzle back pressure. Thus, the supersonic molecular beam pressure and effective helium flow rate at the ion source is independent on the column flow rate and consequently the Cold EI ion source response is column flow rate independent. This feature opens new and unique opportunities with column flow programming to improve the range of compounds amenable for analysis, keep the column clean from matrix compound deposits and speed-up the analysis (Amirav, 2012) [33].

5) Multiple ion sources operation modes and their fast changeover.

In most standard GC-MS (excluding ion traps) systems the replacement of standard EI with CI ion source is lengthy and requires venting and hardware change plus added price. In GC-MS with Cold EI the same flythrough ion source can serve in four modes of operation that are interchangeable via a method change which takes a few seconds or minutes, without any hardware change and without added cost. The fly-through ion source can be operated in the following four modes of Cold EI, Low Electron Energy Cold EI, Classical EI-SMB and Cluster CI. However, the best situation is to be able to work with only one ion source and the fly-through ion source in its Cold EI mode of operation is close to the ideal ion source that outperforms standard EI and CI combined as elaborated throughout this blog post.

6) Temperature programmable transfer line.

GC-MS transfer-lines are currently provided by all vendors without temperature programming capability and thus are typically maintained at the upper GC oven temperature specified in the method such as $300^{\circ} \mathrm{C}$. The Aviv Analytical 5975-SMB GC-MS with Cold EI is uniquely provided with transfer-line temperature programming capability to improve the GC-MS performance in several aspects: A) In the analysis of a mixture of compounds that includes a relatively volatile thermally labile compound the transfer line is maintained at a relatively low initial temperature such as $180^{\circ} \mathrm{C}$ and only after the elution of the thermally labile compound(s) its temperature is increased to prevent peak broadening for the late eluters. A typical example is the analysis of pesticides that include the relatively volatile thermally labile carbamate pesticides (aldicarb, methomyl etc.) as well as less volatile pesticides, and similarly explosives mixtures that include TATP; B) Lower initial transfer-line temperature results in lower PDMS transfer line bleed hence provide lower MS noise and increased sensitivity; C) Every syringe injection includes about $0.5-1 \mu \mathrm{L}$ air in the empty portion of the syringe needle. Consequently, even if the column is cooled during the injection, the pure air that is inevitably injected interacts with the column at its transfer line section, induces PFMS bleeding noise and makes this portion of transfer-line column active with exposed silanol groups. The use of temperature programmable transfer-line significantly reduces this problem.

7) Transfer-line temperature uniformity.

While not specified by any vendor, transfer-lines inherently suffer from having non-uniform temperature along their axis which requires their extra heating to prevent peak broadening which can induce delicate sample decomposition. While the GC side is actively cooled by the GC oven, the ion source side is not fully heated by the transfer-line heater due to limited heat transfer that depends on the design. In fact, since the ion source temperature is insulated from the transfer-line temperature a local cold spot between them is inevitably formed. The transfer-line of the Aviv Analytical 5975-SMB GC-MS with Cold EI is designed with thick aluminum block heater with $28 \mathrm{~mm}$ diameter for effective heat transfer to both sides. In addition, once the sample elutes behind the nozzle it is mixed with $60 \mathrm{ml} / \mathrm{min}$ make-up gas, thereby eliminating peak broadening at the inevitably cooler nozzle even if its temperature is $30^{\circ} \mathrm{C}$ lower. Furthermore, unlike in standard GC-MS transfer-lines, in GC-MS with Cold EI the nozzle is more effectively thermally coupled with the transfer line heater, thereby reducing any cold spot between them. At the GC side, an aluminum jacket transfers the heat from the transfer line heater block up to the column entrance point to minimize any "dynamic cold spot” that can be formed during fast GC oven temperature programs.

8) High temperature GC operation in GC-MS.

In GC-MS with standard EI the current industry standard for upper transfer-line and ion source temperatures is $350^{\circ} \mathrm{C}$. This upper limit restricts the range of low volatility compounds that can be analyzed and in addition, as the ion source temperature is increased, the relative abundances of the molecular ions are exponentially reduced. In the 5975-SMB GC-MS with Cold EI the ion 
source is of a fly-through design hence its temperature (typically $400^{\circ} \mathrm{C}$ ) is irrelevant for the analysis. The transfer-line temperature is limited to $350^{\circ} \mathrm{C}$ although a higher temperature version is offered. On the other hand, the use of short column with increased column flow rate enables the analysis of low volatility sample compounds up to and beyond the mass limit of the 5975 MSD of 1050 amu. However, the penalty for using shorter columns with high column flow rates is in having somewhat reduced chromatographic separating by about a factor of 2 peak capacity per $40^{\circ} \mathrm{C}$ lower elution temperature. As a result, it is beneficial to analyze complex mixtures of stable low volatility compounds using high temperature standard length columns (15 - $60 \mathrm{~m}$ ) with GC oven temperatures up to $420^{\circ} \mathrm{C}$. The 5975-SMB GC-MS with Cold EI enables such high temperature analysis with a backflush $\mathrm{T}$ union device that adds flow rate to the transfer line, and thus with the use of $1 \mathrm{ml} / \mathrm{min}$ column flow rate and $16 \mathrm{ml} / \mathrm{min}$ added make up gas at the transfer line, the GC oven can be heated up to $420^{\circ} \mathrm{C}$ without transfer-line induced peak broadening even if the transfer line is at $350^{\circ} \mathrm{C}$. As a result, low volatility compounds can be analyzed with improved separation.

9) Compatibility with hydrogen or nitrogen carrier gases.

In certain cases the helium supply could be interrupted and one might wish to consider working with hydrogen or nitrogen as the carrier gas. The use of hydrogen with standard EI could lead to the chemical activation of the GC liner and ion source, while the use of nitrogen significantly reduces the ion source ionization yield due to significantly (x7) increased ion source space charge (Amirav, \& Alon, 2012) [34]. Cold EI can uniquely operate with nitrogen as the column carrier gas and hydrogen as make up gas with minimal loss in sensitivity (Amirav, 2013) [35].

10) Ion source robustness.

Ion source robustness is an important feature of any ion source. In standard EI the ion source requires periodic cleaning with an abrasive material to remove polymerized insulating material from its metal surfaces. The Cold EI fly-through ion source is highly robust and requires very little maintenance since $\sim 75 \%$ of the sample compounds are eliminated by the entrance skimmer and from the rest $90 \%$ fly through the ion source thus only $2 \%$ of the sample compounds scatter from the hot $\left(400^{\circ} \mathrm{C}\right)$ and large ion source surface area. In addition, the quadrupole also remains clean as the ion source is separated from the quadrupole mass analyzer by a $90^{\circ}$ ion mirror.

11) Ion source temperature-independent mass spectra.

Obviously users want their GC-MS to provide reproducible mass spectra regardless of the ion source conditions and temperatures. However, the ion source tem- perature strongly affects the obtained standard EI mass spectra. For example, as demonstrated and explained (Amirav et al., 2013) [16], the relative abundance of the molecular ion is exponentially reduced with the ion source temperature. In Cold EI the ion source temperature is irrelevant to the Cold EI mass spectra which are reproducibly obtained. In addition, the Cold EI flythrough ion source is self-cleaned and provides Cold EI MS with little effect of extended use.

12) Bigger GC-MS system pump.

Currently there is a trend of increasing the size of the GC-MS vacuum system pump and older systems with 70 L/s turbo molecular pumps are being replaced by new GC-MS systems that are offered only with bigger $250 \mathrm{~L} / \mathrm{s}$ pumps or split turbo molecular pumps for having differential pumping. In fact, currently all GC-MS vendors sell their systems either with big (performance) turbo molecular pump or with split (differentially pumped) turbo molecular pump. Clearly, with a bigger vacuum pump, pump down time is faster, vacuum background noise is lower and the maximum allowed input flow rate is higher. Thus, the competition on increased OFN specification leads to increased vacuum pump pumping speed despite the added cost. GC-MS with Cold EI includes an additional differentially pumped vacuum chamber with 250 L/s turbo molecular pump, allowing a record high column flow rate (up to $100 \mathrm{ml} / \mathrm{min}$ ) and fully eliminates vacuum background, thus achieving the most from the addition of a differential pumping stage. This trend of using "big" or dual stage pumps reduces the added cost gap between standard GC-MS and GC-MS with Cold EI.

13) Easier and more flexible method development.

For optimal GC-MS operation its method of operation should be tailored to the analysis task. For example, in service GC-MS samples significantly vary in terms of sample types, volatility, thermal stability, and the need for side products identification. As a result, an improved GC-MS system should enable flexible and easy method development, particularly in terms of flow programming and the use of short columns as provided by GC-MS with Cold EI.

14) Demonstration of benefits in challenging applications and new analysis methods.

Every GC-MS vendor praises its system with all the buzzwords and superlatives. All the GC-MS vendors further provide application notes, but these notes mostly demonstrate their system use in standard applications and thus are mostly a "me too" type of statement. However, GC-MS systems should be further evaluated via the availability of application notes and demonstrations of the claimed benefits in the analysis of challenging applications. A large amount of demonstrations of unique and challenging applications are published in the Advanced GC-MS Blog Journal [36]. Furthermore, with Cold EI 
multiple benefits are sometimes combined into the development of unique new analysis methods such as isomer distribution [13], universal pesticide analysis method (Amirav, January 2013) [37], the determination of chemical reaction yields (Amirav et al. 2012) [29] and (Amirav et al., 2013) [28] and a few other methods as described in several blog posts. Finally, the ultimate system test is in its applicability to user's specific applications and goals based on user samples that may serve as the ultimate system performance test and demonstration.

15) Price.

This is the bottom line for many users. The price of GC-MS with standard EI ion source is moderate. The price of GC-MS with Cold EI ion source can be similar to that of standard EI if a fully integrated ion source will be available as can be achieved by a company that will produce a full GC-MS with Cold EI system. The Aviv Analytical 5975-SMB GC-MS with Cold EI serves as an add-on system and thus it about doubles the price of single quad GC-MS but this high price emerges not from the technology itself but rather from the fact that all its components are doubled (vacuum chamber, turbo pump, ion source, transfer line etc).

\subsection{Improved Utilization of Mass Analyzer Specifications}

The mass analyzer specifications clearly belong to "what can be improved in GC-MS". Initially, even though these mass analyzer specifications are not affected by the interface and ion source performance, they are coupled and the improvement of an interface's characteristics can aid or increase the benefits provided by the mass analyzer. Thus, a close examination of these aspects reveals that in fact Cold EI enhances the benefits from and utilization of all the mass analyzer specifications.

1) Improved benefit from extended mass range.

The mass range is a parameter that is specified by all GC-MS vendors. Typically it is from low mass up to and over $1050 \mathrm{amu}$. While such mass range is sufficient for standard GC-MS operation since very few compounds with molecular mass of over 1050 amu can elute from standard GC-MS column, GC-MS with Cold EI can serve for the analysis of much bigger compounds (about doubled mass range) and thus can benefit from improved higher mass range specification. Such large compounds with mass over 1050 amu not only can elute but they also provide useful molecular ions in Cold EI.

2) Improved benefit from higher mass resolution.

Higher mass resolution serves mostly for the reduction of matrix interference. However, in order to benefit from the reduction of matrix interference the sample compound must first elute and as described above (points 12 - 14) the Cold-EI interface enables the analysis of a greater range of compounds. Furthermore, Cold EI also improves the rejection of matrix interference via the enhancement of the molecular ion (Amirav, 2013) [26]. As a result, the effect of high resolution is amplified with Cold EI and the combination of high resolution and enhanced molecular ions can provide the ultimate selectivity and matrix interference rejection.

3) Improved benefit from higher mass accuracy.

High mass accuracy serves for the elucidation of elemental formulas. However, for such service, the sample compound must first elute and than it must exhibit a trustworthy molecular ion. Cold EI excels in comparison with standard EI in both of these aspects thus amplifies the benefits of high mass accuracy. In addition, the TAMI software [5] can combine the high mass accuracy with isotope abundance analysis for obtaining highly improved provision of elemental formula (Alon et al., March 2013) [6].

4) Improved benefit from faster scan speed.

Scan speed is a typical specification highlighted by those vendors who sell GC-MS with time of flight MS. However, the reality is that for fast GC-MS there is no need for scan speeds that are faster than that which is provided by current modern quadrupoles, which is $20,000 \mathrm{amu} / \mathrm{s}$ (40 $\mathrm{Hz}$ for mass range of $50-550 \mathrm{amu}$ ). Only for GCxGC-MS with thermal modulation, in some cases it is desirable to have higher scan speed. However, fast scan speed alone is insufficient for GCxGC-MS since for semi-volatile, low volatility and polar compounds ion source related peek tailing broadens the GC peaks and hampers the GCxGC resolution. As a result, the ion source temperature must be increased to reduce this ion source peak tailing in order to benefit from the fast scan speed and narrow peaks. However, one must remember that an order of magnitude narrower peaks (as claimed for GCxGC-MS by the TOF companies) requires $70^{\circ} \mathrm{C}$ higher ion source temperature than what is needed for standard GC-MS and this $70^{\circ} \mathrm{C}$ higher ion source temperature exponentially reduces the molecular ions and mass spectral information content plus induces ion source degradation for many labile compounds. Cold EI eliminates these problems, provides sub millisecond ion source response time regardless of the sample compounds' volatility or polarity (due to its fly-through operation) and also provides enhanced molecular ions without ion source temperature issues. Thus, since GCxGCMS is all about information, faster scan speed shines brighter with Cold EI as their combination uniquely enables the analysis of very narrow GCxGC peaks of semivolatile compounds.

\section{Discussion}

This post article elaborates on the subject of "what can be improved in GC-MS” and describes sixty two areas of improvements and how they can be obtained via the use 
of the GC-MS with Cold EI with its supersonic molecular beams interface, fly-through ion source and other supporting compatible technologies. Naturally, some improvements are more important than others and for example the extension of the range of compounds amenable for analysis is arguably the most important advantage of GC-MS with Cold EI since it bridges the gap between GC-MS and LC-MS and opens up new areas of analysis and applications. As a result, GC-MS with Cold EI can induce total GC-MS market growth. Quoting Aristotle "The whole is greater than the sum of its parts" and similarly, the combination of so many improvements creates a new and qualitatively superior technology that actually improves every type of analysis. While GC-MS with Cold EI improves challenging analyses it does not impede on any simple method of analysis (compared with standard EI) and its added cost could be negligible in a fully integrated GC-MS with Cold EI. Consequently, GC-MS with Cold EI is destined to become the future GC-MS revolution.

As a good closure of this article, we quote Freeman Dyson from his book "Imagined Worlds":

"New directions in science are launched by new tools much more often than by new concepts. The effect of a concept-driven revolution is to explain old things in new ways. The effect of a tool-driven revolution is to discover new things that have to be explained."

\section{REFERENCES}

[1] A. Amirav, A. Gordin, M. Poliak and A. B. Fialkov, "Gas Chromatography Mass Spectrometry with Supersonic Molecular Beams," Journal of Mass Spectrometry, Vol. 43, No. 2, 2008, pp. 141-163. doi:10.1002/jms.1380

[2] A. Amirav, U. Keshet, T. Alon and A. B. Fialkov, "By how much is the Molecular Ion Enhanced in Cold EI," Advanced GC-MS Blog Journal, 2012.

http://blog.avivanalytical.com/2012/09/how-much-is-mol ecular-ion-enhanced-in.html

[3] A. Amirav and T. Alon, "The Effects of Enhanced Molecular Ions on NIST's Identification Probability,” Advanced GC-MS Blog Journal, 2012. http://blog.avivanalytical.com/2012/09/the-effects-of-enh anced-molecular-ions.html

[4] T. Alon and A. Amirav, "Isotope Abundance Analysis Method and Software for Improved Sample Identification with the Supersonic GC-MS," Rapid Communications in Mass Spectrometry, Vol. 20, No. 17, 2006, pp. 2579-2588. doi:10.1002/rcm.2637

[5] Aviv Analytical Website, “Tal-Aviv Molecule Identifier (TAMI) Software,” 2013. http://www.avivanalytical.com/Isotope-Abundance.aspx

[6] T. Alon and A. Amirav, "Enhancing the Identification Capabilities of EI GC-MS: How Quadrupole GC-MS Can Compete with High Resolution TOF," Advanced GC-MS Blog Journal, 2013. http://blog.avivanalytical.com/2013/03/enhancing-identifi cation-capabilities.html

[7] A. Amirav, "Sample Identification: Which Is a Better Method and Instrument, GC-MS with Its Library Search or LC-HR-MS with the Provision of Elemental Formula?” Advanced GC-MS Blog Journal, 2012.

http://blog.avivanalytical.com/2012/09/sample-identificati on-which-is-better.html

[8] A. Amirav, "Dramatically Improved Hydrocarbon Analysis with the 5975-SMB GC-MS with Cold EI,” Advanced GC-MS Blog Journal, 2012.

http://blog.avivanalytical.com/2012/09/dramatically-impr oved-hydrocarbon.html

[9] A. Gordin, A. Amirav and A. B. Fialkov, "Classical Electron Ionization Mass Spectra in Gas Chromatography/ Mass Spectrometry with Supersonic Molecular Beams," Rapid Communications in Mass Spectrometry, Vol. 22, No. 17, 2008, pp. 2660-2666. doi:10.1002/rcm.3654

[10] A. B. Fialkov and A. Amirav, "Cluster Chemical Ionization for Improved Confidence Level in Sample Identification," Rapid Communications in Mass Spectrometry, Vol. 17, No. 12, 2003, pp. 1326-1338. doi:10.1002/rcm.1057

[11] S. Dagan and A. Amirav, "Cluster Chemical Ionization and Deuterium Exchange Mass Spectrometry in Supersonic Molecular Beams,” Journal of the American Society for Mass Spectrometry, Vol. 7, No. 6, 1996, pp. 550-558. doi:10.1016/1044-0305(96)00017-7

[12] A. B. Fialkov, A. Gordin and A. Amirav, "Hydrocarbons and Fuels Analysis with the Supersonic GC-MS: The Novel Concept of Isomer Abundance Analysis," Journal of Chromatography A, Vol. 1195, No. 1-2, 2008, pp. 127 135. doi:10.1016/j.chroma.2008.04.074

[13] Advanced GC-MS Blog Journal, “Isomer Analysis Article Section,” 2012-2013.

http://blog.avivanalytical.com/search/label/Isomer\%20An alysis

[14] A. B. Fialkov, A. Gordin and A. Amirav, "Extending the Range of Compounds Amenable for Gas Chromatography Mass Spectrometry Analysis,” Journal of Chromatography A, Vol. 991, No. 2, 2003, pp. 217-240. doi:10.1016/S0021-9673(03)00247-4

[15] A. Amirav, R. Seltzer and A. Hefetz, "The Discovery of New Ant Head Compounds via Chromato Probe Sampling and 5975-SMB GC-MS with Cold EI,” Advanced GC-MS Blog Journal, 2013.

http://blog.avivanalytical.com/2013/02/the-discovery-of-n ew-ant-head-compounds.html

[16] A. Amirav, T. Alon and A. B. Fialkov, "Peak TailingLike an Iceberg, It Hides Signal More than Commonly Perceived,” Advanced GC-MS Blog Journal, 2013. http://blog.avivanalytical.com/2013/03/peak-tailing-like-i ceberg-it-hides.html

[17] A. Amirav and S. Dagan, “A Direct Sample Introduction Device for Mass Spectrometry Studies and GC-MS Analysis," European Journal of Mass Spectrometry, Vol. 3, No. 2, 1997, pp. 105-111. doi:10.1255/ejms.27

[18] Aviv Analytical, “The Chromatoprobe,” 2013. http://www.avivanalytical.com/ChromatoProbe.aspx

[19] A. Amirav, “AM2201 Synthetic Cannabis Analysis using 
the 5975-SMB GC-MS with Cold EI,” Advanced GC-MS Blog Journal, 2012.

http://blog.avivanalytical.com/2012/10/am2201-synthetic -cannabis-analysis.html

[20] A. B. Fialkov, M. Morag and A. Amirav, "A Low Thermal Mass Fast Gas Chromatograph and Its Implementation in Fast Gas Chromatography Mass Spectrometry with Supersonic Molecular Beams," Journal of Chromatography A, Vol. 1218, No. 52, 2011, pp. 9375-9383. doi:10.1016/j.chroma.2011.10.053

[21] M. Poliak, A. Gordin and A. Amirav, “Open Probe: A Device for Ultra Fast Electron Ionization Mass Spectrometry Analysis,” Analytical Chemistry, Vol. 82, No. 13, 2010, pp. 5777-5782. doi:10.1021/ac100834h

[22] A. Amirav and T. Alon, "Advanced GC-MS Presentations of Aviv Analytical in Pittcon 2013,” Advanced GC-MS Blog Journal, 2013.

http://blog.avivanalytical.com/2013/03/advanced-gc-ms-p resentations-of-aviv.html

[23] A. B. Fialkov, U. Steiner, S. J. Lehotay and A. Amirav, "Sensitivity and Noise in GC-MS: Achieving Low Limits of Detection for Difficult Analytes," International Journal of Mass Spectrometry, Vol. 260, No. 1, 2007, pp. 3148. doi:10.1016/j.ijms.2006.07.002

[24] A. Amirav, "OFN Sensitivity Specifications-Are They of any Value or Just a Game,” Advanced GC-MS Blog Journal, 2012.

http://blog.avivanalytical.com/2012/08/ofn-sensitivity-spe cifications-are-they.html

[25] M. Kochman, A. Gordin, P. Goldshlag, S. J. Lehotay and A. Amirav, "Fast, High Sensitivity, Multi-Pesticide Analysis of Complex Mixtures with the Supersonic GC-MS," Journal of Chromatography A, Vol. 974, No. 1-2, 2002, pp. 185-212. doi:10.1016/S0021-9673(02)01245-1

[26] A. Amirav, "Selectivity Enhancement for the Reduction of Matrix Interference in GC-MS-Cold EI as an Alternative to MS-MS,” Advanced GC-MS Blog Journal, 2013. http://blog.avivanalytical.com/2013/01/selectivity-enhanc ement-for-reduction.html

[27] A. Amirav, "Drug Impurities Analysis by the Aviv Analytical 5975-SMB GC-MS with Cold EI,” Advanced GC-MS Blog Journal, 2013.

http://blog.avivanalytical.com/2013/02/drug-impurities-a nalysis-by-aviv.html

[28] A. Amirav, A. Gordin, B. Belgorodsky, B. Seeman, M. Gozin and A. B. Fialkov, "Measurement and Optimization of Organic Chemical Reaction Yields by GC-MS with Cold EI,” Advanced GC-MS Blog Journal, 2013. http://blog.avivanalytical.com/2013/01/measurement-andoptimization-of-organic.html

[29] A. Amirav, A. Gordin, Y. Hagooly, S. Rozen, B. Belgorodsky, B. Seemann, H. Marom, M. Gozin and A. B. Fialkov, "Measurement and Optimization of Organic Chemical Reaction Yields by GC-MS with Supersonic Molecular Beams,” Tetrahedron, Vol. 68, 2012, pp. 57935799. doi:10.1016/j.tet.2012.05.031

[30] M. Poliak, M. Kochman and A. Aviv, "Pulsed Flow Modulation Comprehensive Two Dimensional Gas Chromatography,” Journal of Chromatography A, Vol. 1186, No. 1-2, 2008, pp. 189-195. doi:10.1016/j.chroma.2007.09.030

[31] M. Poliak, A. B. Fialkov and A. Amirav, "Pulsed Flow Modulation Two-Dimensional Comprehensive Gas Chromatography Tandem Mass Spectrometry with Supersonic Molecular Beams,” Journal of Chromatography A, Vol. 1210, No. 1, 2008, pp. 108-114. doi:10.1016/j.chroma.2008.09.039

[32] O. Granot and A. Amirav, "LC-MS with Electron Ionization of Cold Molecules in Supersonic Molecular Beams," International Journal of Mass Spectrometry, Vol. 244, No. 1, 2005, pp. 15-28. doi:10.1016/j.ijms.2005.04.003

[33] A. Amirav, "Very Fast Heroin Analysis-The Role of Flow Programming," Advanced GC-MS Blog Journal, 2012.

http://blog.avivanalytical.com/2012/09/very-fast-heroin-a nalysis-role-of-flow.html

[34] A. Amirav and T. Alon, "Helium Shortage and Hydrogen as a Carrier Gas for GC-MS,” Advanced GC-MS Blog Journal, 2012.

http://blog.avivanalytical.com/2012/10/helium-shortage-a nd-hydrogen-as-carrier.html

[35] A. Amirav, "Nitrogen as an Alternative for Helium as a Carrier Gas for 5975-SMB GC-MS with Cold EI,” Advanced GC-MS Blog Journal, 2013.

http://blog.avivanalytical.com/2013/05/nitrogen-as-altern ative-for-helium-as.html

[36] Advanced GC-MS Blog Journal, 2012-2013. http://blog.avivanalytical.com

[37] A. Amirav, "A Universal Method for Pesticide Analysis with the 5975-SMB GC-MS with Cold EI," Advanced GC-MS Blog Journal, 2013.

http://blog.avivanalytical.com/2013/01/a-universal-metho d-for-pesticide.html. 